\title{
Remarks on Homogeneous Al-Salam and Carlitz Polynomials
}

\author{
Jian-Ping Fang \\ School of Mathematical Science, Huaiyin Normal University, Huaian, Jiangsu 223300, China
}

Correspondence should be addressed to Jian-Ping Fang; fjp7402@163.com

Received 2 April 2014; Accepted 4 July 2014; Published 17 July 2014

Academic Editor: Fawang Liu

Copyright (C) 2014 Jian-Ping Fang. This is an open access article distributed under the Creative Commons Attribution License, which permits unrestricted use, distribution, and reproduction in any medium, provided the original work is properly cited.

Several multilinear generating functions of the homogeneous Al-Salam and Carlitz polynomials are derived from $q$-operator. In addition, two interesting relationships of product of this kind of polynomials are obtained.

\section{Introduction}

The Al-Salam and Carlitz polynomials

$$
\phi_{n}^{(a)}(x)=\sum_{k=0}^{n}\left[\begin{array}{l}
n \\
k
\end{array}\right](a ; q)_{k} x^{k}
$$

have been studied by many researchers for a long time. The history of these polynomials may go back to Al-Salam and Carlitz in 1965. Since then, these polynomials have been studied by many mathematicians [1-14].

Recently, Cao [7] used Carlitz's q-operators to study the following homogeneous Al-Salam and Carlitz polynomials:

$$
\phi_{n}^{(a)}(x, y)=\sum_{k=0}^{n}\left[\begin{array}{l}
n \\
k
\end{array}\right](a ; q)_{k} x^{k} y^{n-k}, \quad y \neq 0
$$

and he gave some linear generating functions of them. In this paper, we will research these polynomials by some construction of $q$-operator. With this method, some new multilinear generating functions can be easily derived. Firstly, we give the following three results which originated from the results about $\phi_{n}^{(a)}(x)$ which appeared in $[1,2,5-7,9,10]$.
Theorem 1. If $m, n \in N, \max \{|x X z|,|x Y z|,|y X z|,|y Y z|\}<$ 1 , then

$$
\begin{aligned}
& \sum_{n=0}^{\infty} \phi_{m+n}^{(a)}(x, y) \phi_{n}^{(b)}(X, Y) \frac{z^{n}}{(q ; q)_{n}} \\
& =\frac{y^{m}(q ; q)_{m}(b y X z, a x X z ; q)_{\infty}}{(y Y z, y X z, x X z ; q)_{\infty}} \\
& \quad \times \sum_{k=0}^{\infty} \frac{(x / y)^{k}(a, y Y z, y X z ; q)_{k}}{(q, b y X z, a x X z ; q)_{k}(q ; q)_{m-k}} \\
& \quad \times{ }_{3} \Phi_{2}\left(\begin{array}{c}
a q^{k}, \quad y X z q^{k}, \quad \frac{b X}{Y} \\
b y X z q^{k}, a x X z q^{k}
\end{array} ;, x Y z\right) .
\end{aligned}
$$

Theorem 2. If $m, n \in N, \max \{|x t|,|y t|,|X z|,|Y z|,|y Y \gamma|$, $|y X \gamma|,|x Y \gamma|\}<1$, then

$$
\begin{aligned}
& \sum_{m, n, k=0}^{\infty} \phi_{m+k}^{(a)}(x, y) \phi_{n+k}^{(b)}(X, Y) \frac{t^{m} z^{n} \gamma^{k}}{(q ; q)_{m}(q ; q)_{n}(q ; q)_{k}} \\
& \quad=\frac{(b X z, a x Y \gamma ; q)_{\infty}}{(Y z, X z, y t, y Y \gamma, x Y \gamma ; q)_{\infty}} \times \sum_{m=0}^{\infty} \frac{(y X \gamma)^{m}(b, Y z ; q)_{m}}{(b X z ; q)_{m}}
\end{aligned}
$$




$$
\begin{aligned}
& \times \sum_{k=0}^{\infty} \frac{(x / y)^{k}(a, y t, y Y \gamma ; q)_{k}}{(q, a x Y \gamma ; q)_{k}(q ; q)_{m-k}} \\
& \quad \times{ }_{2} \Phi_{1}\left(\begin{array}{c}
a q^{k}, \quad \begin{array}{c}
y Y \gamma q^{k} \\
a x Y \gamma q^{k}
\end{array} q, x t
\end{array}\right) .
\end{aligned}
$$

Theorem 3 (cf. [7, Equation (1.9)]). If $k, m, n \in N$, $\max \{|x Y z|,|x X z|,|X y z|,|Y y z|\}<1$, then

$$
\begin{aligned}
& \sum_{k=0}^{\infty} \phi_{n+k}^{(a)}(x, y) \phi_{m+k}^{(b)}(X, Y) \frac{z^{k}}{(q ; q)_{k}} \\
& =\frac{y^{n} Y^{m}(q ; q)_{n}(q ; q)_{m}(y b X z, x a X z ; q)_{\infty}}{(y Y z, y X z, x X z ; q)_{\infty}} \\
& \quad \times \sum_{k=0}^{\infty} \frac{(X / Y)^{k}(b, y Y z ; q)_{k}}{(q, y b X z ; q)_{k}(q ; q)_{m-k}} \\
& \quad \times \sum_{j=0}^{\infty} \frac{(x / y)^{j}\left(a, y Y z q^{k}, y X z ; q\right)_{j}}{\left(q, y b X q^{k}, x a X z ; q\right)_{j}(q ; q)_{n-j}} \\
& \quad \times{ }_{3} \Phi_{2}\left(\begin{array}{c}
a q^{j}, \quad y X z q^{j}, \quad \frac{b X}{Y} \\
b y X z q^{k+j}, a x X z q^{j}
\end{array} ;, x Y z q^{k}\right) .
\end{aligned}
$$

Now further using our method, we can deduce more results of multilinear generating functions.

Theorem 4. If $G \in Z^{+}, a_{1}=q^{-G},\left|a_{1} x_{1} / y_{1}\right|<1$, then

$$
\begin{aligned}
& \sum_{m_{1}, m_{2}, m_{3}=0}^{\infty} \phi_{m_{1}+m_{2}+m_{3}}^{\left(a_{1}\right)}\left(x_{1}, y_{1}\right) \phi_{m_{1}+m_{3}}^{\left(a_{2}\right)}\left(x_{2}, y_{2}\right) \\
& \quad \times \phi_{m_{1}+m_{2}}^{\left(a_{3}\right)}\left(x_{3}, y_{3}\right) \frac{t_{1}^{m_{1}} t_{2}^{m_{2}} t_{3}^{m_{3}}}{(q ; q)_{m_{1}}(q ; q)_{m_{2}}(q ; q)_{m_{3}}} \\
& =\frac{\left(a_{1} x_{1} / y_{1}, a_{3} x_{3} t_{2} y_{1}, a_{2} x_{2} t_{1} y_{1} y_{3} ; q\right)_{\infty}}{\left(x_{1} / y_{1}, t_{2} y_{1} y_{3}, t_{3} y_{1} y_{2}, t_{1} y_{1} y_{2} y_{3}, x_{3} t_{2} y_{1}, x_{2} t_{1} y_{1} y_{3} ; q\right)_{\infty}} \\
& \quad \times \sum_{j_{1}=0}^{\infty} \frac{\left(a_{3} ; q\right)_{j_{1}}\left(x_{3} t_{1} y_{1} y_{2}\right)^{j_{1}}(q ; q)_{j_{1}}}{(q ; q)_{j_{1}}} \\
& \quad \times \sum_{j_{2}=0}^{\infty} \frac{\left(x_{2} / y_{2}\right)^{j_{2}}\left(a_{2} ; q\right)_{j_{2}}}{(q ; q)_{j_{2}}(q ; q)_{j_{1}-j_{2}}} \sum_{j_{3}=0}^{\infty} \frac{\left(x_{2} t_{3} y_{1}\right)^{j_{3}}\left(a_{2} q^{j_{2}} ; q\right)_{j_{3}}}{(q ; q)_{j_{3}}}
\end{aligned}
$$

$$
\begin{aligned}
\times \sum_{j_{4}=0}^{\infty} \frac{\left(a_{1}, x_{3} t_{2} y_{1}, x_{2} t_{1} y_{1} y_{3} ; q\right)_{j_{4}}(-1)^{j_{4}} q^{-\left(j_{4}-1\right) j_{4} / 2}}{\left(q, x_{1} /\left(y_{1} q^{j_{4}}\right) ; q\right)_{j_{4}}} \\
\times\left(\frac{x_{1} q^{j_{1}+j_{3}}}{y_{1}}\right)^{j_{4}} \frac{\left(t_{3} y_{1} y_{2} ; q\right)_{j_{2}+j_{4}}\left(t_{2} y_{1} y_{3} ; q\right)_{j_{1}+j_{4}}}{\left(a_{3} x_{3} t_{2} y_{1} ; q\right)_{j_{1}+j_{4}}} \\
\quad \times \frac{\left(t_{1} y_{1} y_{2} y_{3} ; q\right)_{j_{2}+j_{3}+j_{4}}}{\left(a_{2} x_{2} t_{1} y_{1} y_{3} ; q\right)_{j_{2}+j_{3}+j_{4}}},
\end{aligned}
$$

provided that $\max \left\{\left|t_{i} x_{j} y_{l}\right|,\left|t_{1} x_{i} y_{j} y_{l}\right|,\left|t_{1} y_{1} y_{2} y_{3}\right|\right\}<1$, where $1 \leq i \neq j \neq l \leq 3$.

Theorem 5. If $G \in Z^{+}, a_{1}=q^{-G}$, then

$$
\begin{gathered}
\sum_{m_{1}, m_{2}, m_{3}=0}^{\infty} \phi_{m_{1}+m_{2}+m_{3}}^{\left(a_{1}\right)}\left(x_{1}, y_{1}\right) \phi_{m_{1}+m_{2}}^{\left(a_{2}\right)}\left(x_{2}, y_{2}\right) \\
\times \phi_{m_{3}}^{\left(a_{3}\right)}\left(x_{3}, y_{3}\right) \frac{t_{1}^{m_{1}} t_{2}^{m_{2}} t_{3}^{m_{3}}}{(q ; q)_{m_{1}}(q ; q)_{m_{2}}(q ; q)_{m_{3}}} \\
=\frac{\left(a_{1} x_{1} / y_{1}, a_{3} x_{3} t_{3} y_{1}, a_{2} x_{2} t_{2} y_{1} ; q\right)_{\infty}}{\left(x_{1} / y_{1}, t_{3} y_{1} y_{3}, t_{3} y_{1} x_{3}, t_{1} y_{1} y_{2}, t_{2} y_{1} y_{2}, t_{2} x_{2} y_{1} ; q\right)_{\infty}} \\
\times \sum_{j_{1}, j_{2}=0}^{\infty} \frac{\left(a_{2} ; q\right)_{j_{1}}\left(t_{1} x_{2} y_{1}\right)^{j_{1}}(-1)^{j_{2}} q^{-\left(j_{2}-1\right) j_{2} / 2}}{(q ; q)_{j_{1}}} \\
\times \frac{\left(a_{1}, t_{3} y_{3} y_{1}, t_{3} x_{3} y_{1}, t_{2} x_{2} y_{1}, t_{1} y_{2} y_{1} ; q\right)_{j_{2}}}{\left(q, q x_{1} /\left(y_{1} q^{j_{2}}\right), a_{3} t_{2} x_{3} y_{1} ; q\right)_{j_{2}}} \\
\times \frac{\left(t_{2} y_{2} y_{1} ; q\right)_{j_{1}+j_{2}}}{\left(a_{2} t_{2} x_{2} y_{1} ; q\right)_{j_{1}+j_{2}}}\left(\frac{q^{j_{1}} x_{1}}{y_{1}}\right)^{j_{2}},
\end{gathered}
$$

provided that $\max \left\{\left|y_{1} y_{2} t_{1}\right|,\left|y_{1} y_{2} t_{2}\right|, \quad\left|y_{1} y_{3} t_{3}\right|, \quad\left|x_{3} y_{1} t_{3}\right|\right.$, $\left.\left|x_{2} y_{1} t_{1}\right|,\left|x_{1} y_{1} t_{2}\right|,\left|a_{1} x_{1} / y_{1}\right|\right\}<1$.

Theorem 6. If $m_{i} \in N, \max \left\{\left|x_{1}\right|,\left|y_{1} t_{i}\right|,\left|x_{1} t_{i}\right|\right\}<1, i=1,2,3$, then

$$
\begin{aligned}
& \sum_{m_{1}, m_{2}, m_{3}=0}^{\infty} \phi_{m_{1}+m_{2}+m_{3}}^{(a)}\left(x_{1}, y_{1}\right) \\
& \times \frac{t_{1}^{m_{1}} t_{2}^{m_{2}}\left(-t_{3}\right)^{m_{3}} q^{m_{3}\left(m_{3}-1\right) / 2}}{(q ; q)_{m_{1}}(q ; q)_{m_{2}}(q ; q)_{m_{3}}} \\
&= \frac{\left(y_{1} t_{3}, a x_{1} t_{2} ; q\right)_{\infty}}{\left(y_{1} t_{1}, y_{1} t_{2}, x_{1} t_{2} ; q\right)_{\infty}} \\
& \quad \times{ }_{3} \Phi_{2}\left(\begin{array}{c}
a, y_{1} t_{2}, \quad \frac{t_{3}}{t_{1}} ; q, x_{1} t_{1} \\
y_{1} t_{3}, a x_{1} t_{2}
\end{array}\right) .
\end{aligned}
$$


Theorem 7. If $m_{i} \in N, n, G \in Z^{+}, a=q^{-G}, \max \left\{\left|y t_{i}\right|\right.$, $|a x / y|\}<1, i=1,2, \ldots, n$, then

$$
\begin{aligned}
& \sum_{m_{1}, \ldots, m_{n}=0}^{\infty} \phi_{m_{1}+\cdots+m_{n}}^{(a)}(x, y) \frac{t_{1}^{m_{1}} \cdots t_{n}^{m_{n}}}{(q ; q)_{m_{1}} \cdots(q ; q)_{m_{n}}} \\
& =\frac{(a x / y ; q)_{\infty}}{\left(x / y, y t_{1}, y t_{2}, \ldots, y t_{n} ; q\right)_{\infty}} \\
& \quad \times \sum_{j=0}^{\infty} \frac{\left(a, y t_{1}, y t_{2}, \ldots, y t_{n} ; q\right)_{j}(-1)^{j} q^{-(j-1) j / 2}}{\left(q, x /\left(y q^{j}\right) ; q\right)_{j}}\left(\frac{x}{y}\right)^{j} .
\end{aligned}
$$

Theorem 8. If $m_{i} \in N, t_{i} \neq 0, \max \left\{|x|,\left|y t_{i}\right|,\left|x t_{i}\right|\right\}<1, i=$ $1,2,3,4$, then

$$
\begin{aligned}
& \sum_{m_{1}, m_{2}, m_{3}, m_{4}=0}^{\infty} \phi_{m_{1}+m_{2}+m_{3}+m_{4}}^{(a)}(x, y) \\
& \times \frac{\left(-t_{1}\right)^{m_{1}} t_{2}^{m_{2}} t_{3}^{m_{3}}\left(-t_{4}\right)^{m_{4}} q^{m_{1}\left(m_{1}-1\right) / 2} q^{m_{4}\left(m_{4}-1\right) / 2}}{(q ; q)_{m_{1}}(q ; q)_{m_{2}}(q ; q)_{m_{3}}(q ; q)_{m_{4}}} \\
& =\frac{\left(y t_{1}, y t_{4} ; q\right)_{\infty}}{\left(y t_{2}, y t_{3} ; q\right)_{\infty}} \sum_{j=0}^{\infty} \frac{\left(a, t_{1} / t_{2}, y t_{3} ; q\right)_{j}\left(x t_{2}\right)^{j}}{\left(q, y t_{1}, y t_{4} ; q\right)_{j}} \\
& \times{ }_{2} \Phi_{1}\left(\begin{array}{c}
a q^{j}, \\
\frac{t_{4}}{t_{3}} ; q, x t_{3}
\end{array}\right) .
\end{aligned}
$$

Theorem 9. If $m_{i} \in N, \max \left\{\left|y_{i} y t_{i}\right|,\left|x_{i} y t_{i}\right|,\left|a_{0} / x_{0}\right|\right\}<1, i=$ $0,1,2,3$, then

$$
\begin{aligned}
& \sum_{m, m_{1}, m_{2}, m_{3}=0}^{\infty} \phi_{m_{1}+m_{2}+m_{3}+s}^{(a)}(x, y) \phi_{m+r}^{(a)} \\
& \times\left(x_{0}, y_{0}\right) \phi_{m_{1}}^{\left(a_{1}\right)}\left(x_{1}, y_{1}\right) \phi_{m_{2}}^{\left(a_{2}\right)}\left(x_{2}, y_{2}\right) \\
& \times \phi_{m_{3}}^{\left(a_{3}\right)}\left(x_{3}, y_{3}\right) \\
& \times \frac{t^{m_{1}} t_{1}{ }^{m_{1}} t_{2}^{m_{2}} t_{3}^{m_{3}}}{(q ; q)_{m}(q ; q)_{m_{1}}(q ; q)_{m_{2}}(q ; q)_{m_{3}}} \\
&=\left(y^{s} y_{0}^{r}(q ; q)_{r}\left(\frac{a x}{y}, a_{1} y x_{1} t_{1}, a_{2} y x_{2} t_{2}, a_{3} y x_{3} t_{3} ; q\right)_{\infty}\right) \\
& \times\left(\left(\frac{x}{y}, y y_{1} t_{1}, y x_{1} t_{1}, y y_{2} t_{2}, y x_{2} t_{2},\right.\right. \\
&\left.\left.y y_{3} t_{3}, y x_{3} t_{3}, y y_{0} t ; q\right)_{\infty}\right)^{-1}
\end{aligned}
$$

$$
\begin{aligned}
& \times \sum_{j_{1}, j_{2}=0}^{\infty} \frac{\left(x_{0} / y_{0}\right)^{j_{1}}\left(y x_{0} t\right)^{j_{2}}\left(a_{0} ; q\right)_{j_{1}+j_{2}}}{(q ; q)_{j_{1}}(q ; q)_{j_{2}}(q ; q)_{r-j_{1}}} \\
& \times \sum_{j_{3}=0}^{\infty}\left(\left(a, y y_{1} t_{1}, y x_{1} t_{1}, y y_{2} t_{2}, y x_{2} t_{2}, y y_{3} t_{3}, y x_{3} t_{3} ; q\right)_{j_{3}}\right. \\
& \left.\quad \times\left(y y_{0} t ; q\right)_{j_{1}+j_{3}}\right) \\
& \times\left(\left(\frac{q y}{x}, a_{1} y x_{1} t_{1}, a_{2} y x_{2} t_{2}, a_{3} y x_{3} t_{3} ; q\right)_{j_{3}}\right)^{-1} \\
& \times q^{j_{3}\left(j_{2}+s+1\right)},
\end{aligned}
$$

where $t_{0}=t, m_{0}=m$, and $F \in Z^{+}, a_{0}=q^{-F}$.

Theorem 10. If $M_{i} \in Z^{+}, a_{i}=q^{-M_{i}}, x_{i} \neq 0$, and $y_{i} \neq 0$, then

$$
\begin{aligned}
& \sum_{m_{1}, m_{2}, m_{3}, m_{4}=0}^{\infty} \phi_{m_{2}+m_{3}+m_{4}}^{\left(a_{1}\right)}\left(x_{1}, y_{1}\right) \\
& \times \phi_{1+m_{3}+m_{4}}^{\left(a_{2}\right)}\left(x_{2}, y_{2}\right) \phi_{m_{1}+m_{2}+m_{4}}^{\left(a_{3}\right)} \\
& \times\left(x_{3}, y_{3}\right) \phi_{m_{1}+m_{2}+m_{3}}^{\left(a_{4}\right)}\left(x_{4}, y_{4}\right) \\
& \times \frac{\left(-t_{1}\right)^{m_{1}} t_{2}^{m_{2}} t_{3}^{m_{3}}\left(-t_{4}\right)^{m_{4}} q^{m_{1}\left(m_{1}-1\right) / 2} q^{m_{4}\left(m_{4}-1\right) / 2}}{(q ; q)_{m_{1}}(q ; q)_{m_{2}}(q ; q)_{m_{3}}(q ; q)_{m_{4}}} \\
& =\left(\frac{a_{4} x_{4}}{y_{4}}, \frac{a_{3} x_{3}}{y_{3}}, \frac{a_{2} x_{2}}{y_{2}}, \frac{a_{1} x_{1}}{y_{1}}, t_{1} y_{2} y_{3} y_{4}, t_{4} y_{1} y_{2} y_{3} ; q\right)_{\infty} \\
& \times\left(\left(\frac{x_{4}}{y_{4}}, \frac{x_{3}}{y_{3}}, \frac{x_{2}}{y_{2}}, \frac{x_{1}}{y_{1}}, t_{2} y_{1} y_{3} y_{4}, t_{3} y_{1} y_{2} y_{4} ; q\right)_{\infty}\right)^{-1} \\
& \times \sum_{k_{1}, k_{2}, k_{3}, k_{4}=0}^{\infty}\left(\left(a_{4} ; q\right)_{k_{4}}\left(a_{3} ; q\right)_{k_{3}}\left(a_{2} ; q\right)_{k_{2}}\right. \\
& \left.\times\left(a_{1} ; q\right)_{k_{1}} q^{k_{1}+k_{2}+k_{3}+k_{4}}\right) \\
& \times\left(\left(q, \frac{q y_{4}}{x_{4}} ; q\right)_{k_{4}}\left(q, \frac{q y_{3}}{x_{3}} ; q\right)_{k_{3}}\right. \\
& \left.\times\left(q, \frac{q y_{2}}{x_{2}} ; q\right)_{k_{2}}\left(q, \frac{q y_{1}}{x_{1}} ; q\right)_{k_{1}}\right)^{-1} \\
& \times \frac{\left(t_{1} y_{2} y_{3} y_{4} ; q\right)_{k_{2}+k_{3}+k_{4}}\left(t_{4} y_{1} y_{2} y_{3} ; q\right)_{k_{1}+k_{2}+k_{3}}}{\left(t_{2} y_{1} y_{3} y_{4} ; q\right)_{k_{1}+k_{3}+k_{4}}\left(t_{3} y_{1} y_{2} y_{4} ; q\right)_{k_{1}+k_{2}+k_{4}}}
\end{aligned}
$$

provided that $\max \left\{\left|a_{i} x_{i} / y_{i}\right|,\left|t_{i} y_{j} y_{k} y_{l}\right|\right\}<1$, where $1 \leq i \neq j \neq$ $k \neq l \leq 4$. 
Polynomials (2) evidently reduce to the Rogers-Szegö polynomials (cf. [9])

$$
\phi_{n}^{(0)}(x)=H_{n}(x)=\sum_{k=0}^{n}\left[\begin{array}{l}
n \\
k
\end{array}\right] x^{k}
$$

when $a=0$ and $y=1$. And when $y=1$ they reduce to the common Al-Salam and Carlitz polynomials (1) (cf. [9]).

So now we take some special cases for checking.

Let $a=b=0, x=Y=1$ in (3); we have the following.

Corollary 11 (cf. [6, Theorem 1.1] or [9, Equation (4.1)]). If $m, n \in N$, then

$$
\begin{aligned}
\sum_{n=0}^{\infty} H_{m+n}(y) H_{n}(X) \frac{z^{n}}{(q ; q)_{n}} & \\
= & \frac{y^{m}\left(y X z^{2} ; q\right)_{\infty}}{(z, X z, y z, y X ; q)_{\infty}} \\
& \quad \times{ }_{3} \Phi_{1}\left(\begin{array}{c}
q^{-m}, \quad y z, \\
y X z^{2}
\end{array} \quad y X z ; q, \frac{q^{m}}{y}\right),
\end{aligned}
$$

provided that $\max \{|z|,|y z|,|X z|,|y X z|\}<1$.

Remark 12 (from [6, Equation (3.1)]). We know that Corollary 11 is equivalent to Theorem 1.1 given in [6]. And if we take $m=0$ and $y=Y=1$, (3) turns to [9, Equation (1.2)].

Taking $a=b=0, x=Y=1$ in (4), we have the following.

Corollary 13 (cf. [6, Theorem 1.2] or [9, Equation (1.3)]). If $m, n \in N$, then

$$
\begin{aligned}
& \sum_{m, n, k=0}^{\infty} H_{m+k}(y) H_{n+k}(X) \frac{t^{m} z^{n} \gamma^{k}}{(q ; q)_{m}(q ; q)_{n}(q ; q)_{k}} \\
& =\frac{(y t \gamma, y X z \gamma ; q)_{\infty}}{(t, z, X z, y t, \gamma, y \gamma, y X \gamma ; q)_{\infty}} \\
& \times{ }_{3} \Phi_{2}\left(\begin{array}{ccc}
z, & y t, & y \gamma \\
y t \gamma, & y X z \gamma
\end{array} ; q, X \gamma\right),
\end{aligned}
$$

provided that $\max \{|t|,|z|,|\gamma|,|y t|,|y \gamma|,|X z|,|X \gamma|,|y X \gamma|\}<$ 1.

Remark 14. Using Hall's transformation [15, Equation (3.2.7)]

$$
\begin{aligned}
& { }_{3} \Phi_{2}\left(\begin{array}{ccc}
a, & b, & c \\
& d, & e
\end{array} ; q, \frac{d e}{(a b c)}\right)
\end{aligned}
$$

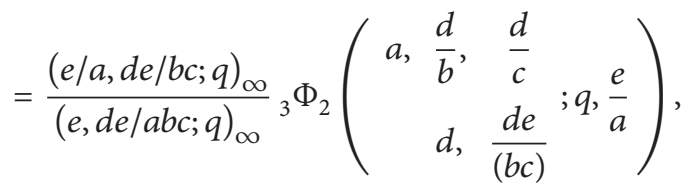

we find that Corollary 13 is equivalent to Theorem 1.2 given in [6]. And if we take $y=Y=1$, then, with simplifying, (4) turns to [9, Equation (3.5)].
Taking $a=b=0$ and $x=X=1$ in (5), we have the following.

Corollary 15 (cf. [6, Theorem 1.3] or [9, Equation (1.4)]). If $m, n, k \in N$, then

$$
\begin{aligned}
& \sum_{k=0}^{\infty} H_{n+k}(y) H_{m+k}(Y) \frac{z^{k}}{(q ; q)_{k}} \frac{\left(y Y z^{2} ; q\right)_{\infty}}{(z, y z, Y z, y Y z ; q)_{\infty}} \\
& \times \sum_{k=0}^{m} \sum_{j=0}^{n}\left[\begin{array}{c}
m \\
k
\end{array}\right]\left[\begin{array}{c}
m \\
j
\end{array}\right] \frac{Y^{m-k} y^{n-j}(y Y z ; q)_{k+j}}{\left(y Y z^{2} ; q\right)_{k+j}} \\
& \times(Y z ; q)_{k}(y z ; q)_{j},
\end{aligned}
$$

provided that $\max \{|z|,|y z|,|Y z|,|y Y z|\}<1$.

The rest of this paper is organized as follows. In Section 2, we will give some notations and lemmas. In Section 3, we give the proofs of theorems. Section 4 describes the relationship between $\Psi_{n}\left(a, b ; x_{1}, y_{1} ; z\right)$ (cf. [4, Equation (2.2)]) and $\phi_{n}^{(a)}(x, y)$. In addition, an interesting relationship between the polynomial multiplications is given.

\section{Notations and Some Lemmas}

In this paper, we apply the standard notations that follow from [15] and assume that $0<|q|<1$; the $q$-shifted factorial and its compact factorials are defined, respectively, by

$$
\begin{aligned}
(a ; q)_{0} & =1 ; \\
(a ; q)_{n} & =\prod_{k=0}^{n-1}\left(1-a q^{k}\right), \\
(a ; q)_{\infty} & =\prod_{k=0}^{\infty}\left(1-a q^{k}\right)
\end{aligned}
$$

and $\left(a_{1}, \ldots, a_{m} ; q\right)_{n}=\left(a_{1} ; q\right)_{n}, \ldots,\left(a_{m} ; q\right)_{n}$, where $n=$ $0,1, \ldots, \infty$. And we use $N$ to denote the set of nonnegative integers and $Z^{+}$to denote the set of positive integers.

For any complex number $\alpha$, we have

$$
(a ; q)_{\alpha}=\frac{(a ; q)_{\infty}}{\left(a q^{\alpha} ; q\right)_{\infty}} .
$$

The $q$-binomial coefficient and the $q$-binomial theorem are given by

$$
\left[\begin{array}{l}
n \\
k
\end{array}\right]=\frac{(q ; q)_{n}}{(q ; q)_{k}(q ; q)_{n-k}}, \quad \sum_{m=0}^{\infty} \frac{(a ; q)_{m}}{(q ; q)_{m}} y^{m}=\frac{(a y ; q)_{\infty}}{(y ; q)_{\infty}},
$$

respectively. 
The basic hypergeometric series ${ }_{r} \Phi_{s}$ is defined by

$$
\begin{aligned}
{ }_{r} \Phi_{s}\left(\begin{array}{c}
a_{1}, \ldots, a_{r} \\
b_{1}, \ldots, b_{s}
\end{array} q, x\right) \\
=\sum_{n=0}^{\infty} \frac{\left(a_{1}, a_{2}, \ldots, a_{r} ; q\right)_{n}}{\left(q, b_{1}, \ldots, b_{s} ; q\right)_{n}} \\
\quad \times\left[(-1)^{n} q^{n(n-1) / 2}\right]^{1+s-r} x^{n} .
\end{aligned}
$$

The $q$-derivative operator $D_{q}$ and $q$-shifted operator $\eta$ (cf. $[1,6,7,9,10,16-23])$, acting on the variable $x$, are defined by

$$
\begin{gathered}
D_{q}\{f(x)\}=\frac{f(x)-f(x q)}{x}, \\
\eta\{f(x)\}=f(x q) .
\end{gathered}
$$

The $q$-Leibnitz rule for the product of two functions (cf. [17-25]) is given by

$$
D_{q}^{n}\{f(x) g(x)\}=\sum_{k=0}^{n}\left[\begin{array}{l}
n \\
k
\end{array}\right] q^{k(k-n)} D_{q}^{k}\{f(x)\} D_{q}^{n-k}\left\{g\left(x q^{k}\right)\right\} .
$$

From definition, we can easily obtain $D_{q}=x^{-1}(1-\eta)$; by mathematical induction, we have the following proposition.

Proposition 16 (cf. [20, Equation (6)]). If $f(x)$ is any analytical function, then

$$
D_{q}^{n}\{f(x)\}=x^{-n} \sum_{k=0}^{n} \frac{\left(q^{-n} ; q\right)_{k}}{(q ; q)_{k}}(q \eta)^{k}\{f(x)\} .
$$

In $[19,20]$, we had established the following $q$-operator structure:

$$
{ }_{1} \Phi_{0}\left(\begin{array}{c}
b \\
-
\end{array} q, c D_{q}\right)=\sum_{n=0}^{\infty} \frac{(b ; q)_{n}\left(c D_{q}\right)^{n}}{(q ; q)_{n}}
$$

For convenient, we use $F\left(a ; x D_{q}\right)$ y to denote the operator ${ }_{1} \Phi_{0}\left({ }_{-}^{a} ; q, x D_{q}\right)$ acting on variable $y$. From this definition we can easily get the following Lemma.

Lemma 17. One has

$$
\phi_{n}^{(a)}(x, y)=F\left(a ; x D_{q}\right)_{y}\left\{y^{n}\right\}
$$

Proof. For

$$
D_{q}^{(k)}\left\{y^{n}\right\}=\frac{(q ; q)_{n}}{(q ; q)_{n-k}} y^{n-k}
$$

we have

$$
\begin{aligned}
& F\left(a ; x D_{q}\right)_{y}\left\{y^{n}\right\} \\
& =\sum_{k=0}^{\infty} \frac{(a ; q)_{k} x^{k}}{(q ; q)_{k}} \frac{(q ; q)_{n}}{(q ; q)_{n-k}} y^{n-k} \\
& =\sum_{k=0}^{n}\left[\begin{array}{l}
n \\
k
\end{array}\right](a ; q)_{k} x^{k} y^{n-k},
\end{aligned}
$$

which completes the proof.
It is the reason why we employ this operator to study the properties of homogeneous Al-Salam and Carlitz polynomials.

From Proposition 16, we may easily obtain the following identity.

Lemma 18. If $G \in Z^{+}, a=q^{-G},|a x / y|<1$ and $f(x)$ is any analytical function, then

$$
\begin{aligned}
& F\left(a ; x D_{q}\right)_{y}\{f(y)\} \\
&=\frac{(a x / y ; q)_{\infty}}{(x / y ; q)_{\infty}} \sum_{k=0}^{\infty} \frac{(-1)^{k}(a ; q)_{k}(x / y)^{k}}{\left(q, x /\left(y q^{k}\right) ; q\right)_{k}} \\
& \times q^{-k(k-1) / 2} f\left(y q^{k}\right) .
\end{aligned}
$$

Proof. Applying Proposition 16, LHS of Lemma 18 comes to

$$
\begin{aligned}
& \sum_{n=0}^{\infty} \frac{(a ; q)_{n}(x / y)^{n}}{(q ; q)_{n}} \\
& \times \sum_{k=0}^{n} \frac{(q ; q)_{n} q^{k}}{(q ; q)_{k}(q ; q)_{n-k}}(-1)^{k} q^{k(k-1) / 2-n k} f\left(y q^{k}\right) \\
&=\sum_{k=0}^{\infty} \frac{(-1)^{k}(a ; q)_{k}(x / y)^{k}}{(q ; q)_{k}} q^{-k(k-1) / 2} f\left(y q^{k}\right) \\
& \quad \times \sum_{n=0}^{\infty} \frac{\left(a q^{k} ; q\right)_{n}}{(q ; q)_{n}}\left(\frac{x}{y q^{k}}\right)^{n} \\
&=\sum_{k=0}^{\infty} \frac{(-1)^{k}(a ; q)_{k}(x / y)^{k}}{(q ; q)_{k}} q^{-k(k-1) / 2} f\left(y q^{k}\right) \\
& \quad \times \frac{(a x / y ; q)_{\infty}}{\left(x /\left(y q^{k}\right) ; q\right)_{\infty}} \times \frac{(a x / y ; q)_{\infty} \sum_{\infty}^{\infty} \frac{(-1)^{k}(a ; q)_{k}(x / y)^{k}}{\left(q, x /\left(y q^{k}\right) ; q\right)_{k}}}{(x / y ; q)_{\infty}} \times q^{-k(k-1) / 2} f\left(y q^{k}\right) . \\
&= \\
&=
\end{aligned}
$$

We complete the proof.

Remark 19. To calculate the inner sum of the above, under the condition $a=q^{-G}, q$-binomial theorem is usable.

Lemma 20 (cf. [20, Lemma 1]). If $|s c|<1$, $|t c|<1$, then

$$
\begin{aligned}
& F\left(b ; c D_{q}\right)_{a}\left\{\frac{(a \omega ; q)_{\infty}}{(a s, a t ; q)_{\infty}}\right\} \\
& \quad=\frac{(a \omega, b c t ; q)_{\infty}}{(a s, a t, c t ; q)_{\infty}}{ }_{3} \Phi_{2}\left(\begin{array}{rl}
b, & \left.a t, \frac{\omega}{s} ; q, s c\right) . \\
a \omega, b c t
\end{array}\right)
\end{aligned}
$$


Proof. LHS of Lemma 20 equates to

$$
\begin{aligned}
& \sum_{n=0}^{\infty} \frac{(b ; q)_{n} c^{n}}{(q ; q)_{n}} \sum_{k=0}^{n}\left[\begin{array}{l}
n \\
k
\end{array}\right] q^{k(k-n)} D_{q}^{k}\left\{\frac{(a \omega ; q)_{\infty}}{(a s ; q)_{\infty}}\right\} \\
& \times D_{q}^{n-k}\left\{\frac{1}{\left(a t q^{k} ; q\right)_{\infty}}\right\} \\
& =\sum_{n=0}^{\infty} \frac{(b ; q)_{n} c^{n}}{(q ; q)_{n}} \\
& \times \sum_{k=0}^{n}\left[\begin{array}{l}
n \\
k
\end{array}\right] q^{k(k-n)} \frac{s^{k}(\omega / s ; q)_{k}\left(a \omega q^{k} ; q\right)_{\infty}}{(a s ; q)_{\infty}} \frac{\left(t q^{k}\right)^{n-k}}{\left(a t q^{k} ; q\right)_{\infty}} \\
& =\frac{(a \omega ; q)_{\infty}}{(a s, a t ; q)_{\infty}} \sum_{k=0}^{\infty} \frac{(a t, \omega / s ; q)_{k}}{(q, a \omega ; q)_{k}} s^{k} \\
& \times \sum_{n=k}^{\infty} \frac{(b ; q)_{n}}{(q ; q)_{n-k}} c^{n} t^{n-k} \\
& =\frac{(a \omega ; q)_{\infty}}{(a s, a t ; q)_{\infty}} \sum_{k=0}^{\infty} \frac{(a t, \omega / s ; q)_{k}}{(q, a \omega ; q)_{k}}(s c)^{k} \\
& \times \sum_{m=0}^{\infty} \frac{(b ; q)_{m+k}}{(q ; q)_{m}}(t c)^{m} \\
& =\frac{(a \omega ; q)_{\infty}}{(a s, a t ; q)_{\infty}} \sum_{k=0}^{\infty} \frac{(a t, \omega / s, b ; q)_{k}}{(q, a \omega ; q)_{k}}(s c)^{k} \\
& \times \sum_{m=0}^{\infty} \frac{\left(b q^{k} ; q\right)_{m}}{(q ; q)_{m}}(t c)^{m} \\
& =\frac{(a \omega ; q)_{\infty}}{(a s, a t ; q)_{\infty}} \sum_{k=0}^{\infty} \frac{(a t, \omega / s, b ; q)_{k}}{(q, a \omega ; q)_{k}}(s c)^{k} \frac{\left(b c t q^{k} ; q\right)_{\infty}}{(c t ; q)_{\infty}} \\
& =\frac{(a \omega, b c t ; q)_{\infty}}{(a s, a t, c t ; q)_{\infty}}{ }_{3} \Phi_{2}\left(\begin{array}{rrr}
b, & a t, & \frac{\omega}{s} \\
a \omega, & b c t
\end{array} ;, s c\right) .
\end{aligned}
$$

We complete the proof.

The special cases of Lemma 20 as taking $t=0, \omega=t=0$, and $\omega=0$, respectively, will be frequently used.

Lemma 21. For $|s c|<1$,

$$
F\left(b ; c D_{q}\right)_{a}\left\{\frac{(a \omega ; q)_{\infty}}{(a s ; q)_{\infty}}\right\}=\frac{(a \omega ; q)_{\infty}}{(a s ; q)_{\infty}}{ }_{2} \Phi_{1}\left(b, \frac{\omega}{s} ; q, s c\right)
$$

Lemma 22. If $|s c|<1$, then

$$
F\left(b ; c D_{q}\right)_{a}\left\{\frac{1}{(a s ; q)_{\infty}}\right\}=\frac{(b c s ; q)_{\infty}}{(a s, c s ; q)_{\infty}} .
$$

Lemma 23. For $|s c|<1$, $|t c|<1$,

$$
\begin{aligned}
& F\left(b ; c D_{q}\right)_{a}\left\{\frac{1}{(a s, a t ; q)_{\infty}}\right\} \\
& =\frac{(b c t ; q)_{\infty}}{(a s, a t, c t ; q)_{\infty}}{ }_{2} \Phi_{1}\left(\begin{array}{cc}
b, & a t \\
b c t
\end{array} ; q, s c\right) .
\end{aligned}
$$

Throughout this paper, we also often use the following property.

Lemma 24. If $|s c|<1$, $|t c|<1$, then

$$
\begin{aligned}
F\left(b ; c D_{q}\right)_{a}\left\{\frac{a^{M}(a \omega ; q)_{\infty}}{(a s, a t ; q)_{\infty}}\right\} \\
=\frac{a^{M}(q ; q)_{M}(a \omega, b c t ; q)_{\infty}}{(a s, a t, c t ; q)_{\infty}} \\
\times \sum_{k=0}^{\infty} \frac{(c / a)^{k}(b, a s, a t ; q)_{k}}{(q, a \omega, b c t ; q)_{k}(q ; q)_{M-k}} \\
\quad \times{ }_{3} \Phi_{2}\left(\begin{array}{c}
b q^{k}, a t q^{k}, \\
\frac{\omega}{s}
\end{array} \quad a \omega q^{k}, b c t q^{k}\right.
\end{aligned}
$$

Proof. We find that LHS of Lemma 24 equates to

$$
\begin{aligned}
\sum_{n=0}^{\infty} \frac{(b ; q)_{n} c^{n}}{(q ; q)_{n}} \sum_{k=0}^{n} & {\left[\begin{array}{l}
n \\
k
\end{array}\right] q^{k(k-n)} D_{q}^{k}\left\{a^{M}\right\} } \\
& \times D_{q}^{n-k}\left\{\frac{\left(a \omega q^{k} ; q\right)_{\infty}}{\left(a s q^{k}, a t q^{k} ; q\right)_{\infty}}\right\}
\end{aligned}
$$$$
=\sum_{k=0}^{\infty} \frac{(q ; q)_{M} a^{M-k}}{(q ; q)_{k}(q ; q)_{M-k}}
$$$$
\times \sum_{n=k}^{\infty} \frac{(b ; q)_{n} c^{n} q^{k(k-n)}}{(q ; q)_{n-k}} D_{q}^{n-k}\left\{\frac{\left(a \omega q^{k} ; q\right)_{\infty}}{\left(a s q^{k}, a t q^{k} ; q\right)_{\infty}}\right\}
$$$$
=a^{M}(q ; q)_{M} \sum_{k=0}^{\infty} \frac{(c / a)^{k}(b ; q)_{k}}{(q ; q)_{k}(q ; q)_{M-k}} F\left(b q^{k} ;\left(\frac{c}{q^{k}}\right) D_{q}\right)
$$

$$
\times\left\{\frac{\left(a \omega q^{k} ; q\right)_{\infty}}{\left(a s q^{k}, a t q^{k} ; q\right)_{\infty}}\right\}
$$




$$
\begin{aligned}
& =\frac{a^{M}(q ; q)_{M}(a \omega, b c t ; q)_{\infty}}{(a s, a t, c t ; q)_{\infty}} \\
& \times \sum_{k=0}^{\infty} \frac{(c / a)^{k}(b, a s, a t ; q)_{k}}{(q, a \omega, b c t ; q)_{k}(q ; q)_{M-k}}
\end{aligned}
$$

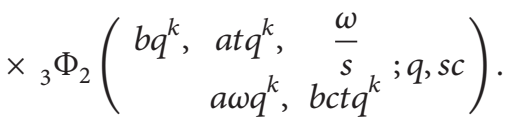

This completes the proof.

\section{Proof of Theorems}

Proof of Theorem 1. LHS of (3) is equal to

$$
\begin{aligned}
\sum_{n=0}^{\infty} F\left(a ; x D_{q}\right)_{y}\left\{y^{(m+n)}\right\} F\left(b ; X D_{q}\right)_{Y}\left\{Y^{(n)}\right\} \frac{z^{n}}{(q ; q)_{n}} & \\
= & F\left(a ; x D_{q}\right)_{y} F\left(b ; X D_{q}\right)_{Y}\left\{\frac{y^{m}}{(Y y z ; q)_{\infty}}\right\} \\
= & F\left(a ; x D_{q}\right)_{y}\left\{\frac{y^{m}(y b X z ; q)_{\infty}}{(y Y z, y X z ; q)_{\infty}}\right\} \\
= & \frac{y^{m}(q ; q)_{m}(b y X z, a x X z ; q)_{\infty}}{(y Y z, y X z, x X z ; q)_{\infty}} \\
& \times \sum_{k=0}^{\infty} \frac{(x / y)^{k}(a, y Y z, y X X z ; q)_{k}}{(q, b y, a x X z ; q)_{k}(q ; q)_{m-k}} \\
& \times{ }_{3} \Phi_{2}\left(\begin{array}{c}
a q^{k}, \quad y X z q^{k}, \quad \frac{b X}{Y} \\
b y X z q^{k}, a x X z q^{k}
\end{array} ;, x Y z\right) .
\end{aligned}
$$

We complete the proof.

Proof of Theorem 2. LHS of (4) is equal to

$$
\begin{aligned}
F\left(a ; x D_{q}\right)_{y}\left\{\frac{1}{(y t: q)_{\infty}} F\left(b ; X D_{q}\right)_{Y}\left\{\frac{1}{(Y y \gamma, Y z ; q)_{\infty}}\right\}\right\} \\
=\frac{\left(b X z ; q_{\infty}\right)}{(Y z, X z ; q)_{\infty}} \sum_{m=0}^{\infty} \frac{(b, Y z ; q)_{m}(X \gamma)^{m}}{(q ; b X z)_{m}} \\
\times F\left(a ; x D_{q}\right)_{y}\left\{\frac{y^{m}}{(y t, y Y \gamma: q)_{\infty}}\right\} ;
\end{aligned}
$$

then using Lemma 24 and simplifying, we can get the desired result.
Proof of Theorem 3. LHS of (5) is equal to

$$
\begin{aligned}
F\left(a ; x D_{q}\right)_{y}\left\{y^{n} F\left(b ; X D_{q}\right)_{Y}\left\{\frac{Y^{m}}{(Y y z ; q)_{\infty}}\right\}\right\} \\
=Y^{m}(q ; q)_{m} \sum_{k=0}^{\infty} \frac{(X / Y)^{k}(b ; q)_{k}}{(q ; q)_{k}(q ; q)_{m-k}} F\left(a ; x D_{q}\right)_{y} \\
\quad \times\left\{\frac{y^{n}\left(y b X z q^{k} ; q\right)_{\infty}}{\left(y Y z q^{k}, y X z ; q\right)_{\infty}}\right\} \\
=\frac{y^{n} Y^{m}(q ; q)_{n}(q ; q)_{m}(y b X z, x a X z ; q)_{\infty}}{(y Y z, y X z, x X z ; q)_{\infty}} \\
\times \sum_{k=0}^{\infty} \frac{(X / Y)^{k}(b, y Y z ; q)_{k}}{(q, y b X z ; q)_{k}(q ; q)_{m-k}} \\
\quad \times \sum_{j=0}^{\infty} \frac{(x / y)^{j}\left(a, y Y z q^{k}, y X z ; q\right)_{j}}{\left(q, y b X z q^{k}, x a X z ; q\right)_{j}(q ; q)_{n-j}} \\
\quad \times{ }_{3} \Phi_{2}\left(\begin{array}{c}
a q^{j}, \quad y X z q^{j}, \quad \frac{b X}{Y} \\
b y X z q^{k+j}, a x X z q^{j}
\end{array} q, x Y z q^{k}\right) .
\end{aligned}
$$

This completes the proof.

Proof of Theorem 4. LHS of (6) is equal to

$$
\begin{aligned}
\sum_{m_{1}, m_{2}, m_{3}=0}^{\infty} & F\left(a_{1} ; x_{1} D_{q}\right)_{y_{1}}\left\{y_{1}^{m_{1}+m_{2}+m_{3}}\right\} F\left(a_{2} ; x_{2} D_{q}\right)_{y_{2}} \\
& \times\left\{y_{2}^{m_{1}+m_{3}}\right\} F\left(a_{3} ; x_{3} D_{q}\right)_{y_{3}}\left\{y_{3}^{m_{1}+m_{2}}\right\} \\
& \times \frac{t_{1}^{m_{1}} t_{2}^{m_{2}} t_{3}^{m_{3}}}{(q ; q)_{m_{1}}(q ; q)_{m_{2}}(q ; q)_{m_{3}}} \\
= & F\left(a_{1} ; x_{1} D_{q}\right)_{y_{1}} F\left(a_{2} ; x_{2} D_{q}\right)_{y_{2}} F\left(a_{3} ; x_{3} D_{q}\right)_{y_{3}}
\end{aligned}
$$

$$
\begin{aligned}
& \times\left\{\frac{1}{\left(t_{1} y_{1} y_{2} y_{3}, t_{2} y_{1} y_{3}, t_{3} y_{1} y_{2} ; q\right)_{\infty}}\right\} \\
= & F\left(a_{1} ; x_{1} D_{q}\right)_{y_{1}} F\left(a_{2} ; x_{2} D_{q}\right)_{y_{2}} \\
& \times\left\{\frac{1}{\left(t_{3} y_{1} y_{2} ; q\right)_{\infty}} F\left(a_{3} ; x_{3} D_{q}\right)_{y_{3}}\right. \\
& \left.\times\left\{\frac{1}{\left(t_{1} y_{1} y_{2} y_{3}, t_{2} y_{1} y_{3} ; q\right)_{\infty}}\right\}\right\} \\
= & F\left(a_{1} ; x_{1} D_{q}\right)_{y_{1}} F\left(a_{2} ; x_{2} D_{q}\right)_{y_{2}} \\
& \times\left\{\frac{\left(a_{3} x_{3} t_{2} y_{1} ; q\right)_{\infty}}{\left(t_{2} y_{1} y_{3}, x_{3} t_{2} y_{1} ; q\right)_{\infty}} \sum_{j_{1}=0}^{\infty} \frac{\left(a_{3}, t_{2} y_{1} y_{3} ; q\right)_{j_{1}}}{\left(q, a_{3} x_{3} t_{2} y_{1} ; q\right)_{j_{1}}}\right.
\end{aligned}
$$

$$
\left.\times \frac{\left(x_{3} t_{1} y_{1} y_{2}\right)^{j_{1}}}{\left(t_{3} y_{1} y_{2}, t_{1} y_{1} y_{2} y_{3} ; q\right)_{\infty}}\right\}
$$




$$
\begin{aligned}
&= F\left(a_{1} ; x_{1} D_{q}\right)_{y_{1}} \\
& \times\left\{\frac{\left(a_{3} x_{3} t_{2} y_{1} ; q\right)_{\infty}}{\left(t_{2} y_{1} y_{3}, x_{3} t_{2} y_{1} ; q\right)_{\infty}} \sum_{j_{1}=0}^{\infty} \frac{\left(a_{3}, t_{2} y_{1} y_{3} ; q\right)_{j_{1}}}{\left(q, a_{3} x_{3} t_{2} y_{1} ; q\right)_{j_{1}}}\right. \\
& \times \sum_{j_{1}=0}^{\infty} \frac{\left(a_{3} ; q\right)_{j_{1}}\left(x_{3} t_{1} y_{2}\right)^{j_{1}}(q ; q)_{j_{1}}}{(q ; q)_{j_{1}}} \\
&\left.\times \sum_{j_{2}=0}^{\infty} \frac{\left(x_{2} / y_{2}\right)^{j_{2}}\left(a_{2} ; q\right)_{j_{2}}}{(q ; q)_{j_{2}}(q ; q)_{j_{1}-j_{2}}}\left\{\frac{\left(x_{3} t_{1} y_{1} y_{2}\right)^{j_{1}}}{\left(t_{3} y_{1} y_{2}, t_{1} y_{1} y_{2} y_{3} ; q\right)_{\infty}}\right\}\right\} \\
& \times \sum_{j_{3}=0}^{\infty} \frac{\left(x_{2} t_{3}\right)^{j_{3}}\left(a_{2} q^{j_{2}} ; q\right)_{j_{3}}}{(q ; q)_{j_{3}}} F\left(a_{1} ; x_{1} D_{q}\right)_{y_{1}} \\
& \times\left\{\left(t_{2} y_{1} y_{3} q^{j_{1}}, t_{3} y_{1} y_{2} q^{j_{2}}, t_{1} y_{1} y_{2} y_{3} q^{j_{2}+j_{3}},\right.\right. \\
&\left.y_{1}^{j_{1}+j_{3}}\left(a_{3} x_{3} t_{2} y_{1} q^{j_{1}}, a_{2} x_{2} t_{1} y_{1} y_{3} q^{j_{2}+j_{3}} ; q\right)_{\infty}\right) \\
&\left.\left.\left.x_{2} t_{1} y_{1} y_{3} ; q\right)_{\infty}\right)^{-1}\right\} .
\end{aligned}
$$

Setting

$$
\begin{gathered}
f\left(y_{1}\right)=\left(y_{1}^{j_{1}+j_{3}}\left(a_{3} x_{3} t_{2} y_{1} q^{j_{1}}, a_{2} x_{2} t_{1} y_{1} y_{3} q^{j_{2}+j_{3}} ; q\right)_{\infty}\right) \\
\times\left(\left(t_{2} y_{1} y_{3} q^{j_{1}}, t_{3} y_{1} y_{2} q^{j_{2}}, t_{1} y_{1} y_{2} y_{3} q^{j_{2}+j_{3}},\right.\right. \\
\left.\left.\quad x_{3} t_{2} y_{1}, x_{2} t_{1} y_{1} y_{3} ; q\right)_{\infty}\right)^{-1},
\end{gathered}
$$

then by applying Lemma 18 and simplifying, we get

$$
\begin{aligned}
& F\left(a_{1} ; x_{1} D_{q}\right)_{y_{1}}\left\{f\left(y_{1}\right)\right\} \\
& =\left(y_{1}^{j_{1}+j_{3}}\left(\frac{a_{1} x_{1}}{y_{1}}, a_{3} x_{3} t_{2} y_{1}, a_{2} x_{2} t_{1} y_{1} y_{3} ; q\right)_{\infty}\right) \\
& \quad \times\left(\left(\frac{x_{1}}{y_{1}}, t_{2} y_{1} y_{3}, t_{3} y_{1} y_{2}, t_{1} y_{1} y_{2} y_{3},\right.\right.
\end{aligned}
$$$$
\left.\left.x_{3} t_{2} y_{1}, x_{2} t_{1} y_{1} y_{3} ; q\right)_{\infty}\right)^{-1}
$$

$$
\begin{aligned}
\times \sum_{j_{4}=0}^{\infty} & \frac{\left(a_{1}, x_{3} t_{2} y_{1}, x_{2} t_{1} y_{1} y_{3} ; q\right)_{j_{4}}(-1)^{j_{4}} q^{-\left(j_{4}-1\right) j_{4} / 2}}{\left(q, x_{1} /\left(y_{1} q^{j_{4}}\right) ; q\right)_{j_{4}}} \\
& \times\left(\frac{x_{1} q^{j_{1}+j_{3}}}{y_{1}}\right)^{j_{4}} \frac{\left(t_{3} y_{1} y_{2} ; q\right)_{j_{2}+j_{4}}\left(t_{2} y_{1} y_{3} ; q\right)_{j_{1}+j_{4}}}{\left(a_{3} x_{3} t_{2} y_{1} ; q\right)_{j_{1}+j_{4}}} \\
& \times \frac{\left(t_{1} y_{1} y_{2} y_{3} ; q\right)_{j_{2}+j_{3}+j_{4}}}{\left(a_{2} x_{2} t_{1} y_{1} y_{3} ; q\right)_{j_{2}+j_{3}+j_{4}}} ;
\end{aligned}
$$

substituting it into (41), we complete the theorem.

Proof of Theorem 5. LHS of (7) is equal to

$$
\begin{aligned}
& F\left(a_{1} ; x_{1} D_{q}\right)_{y_{1}} F\left(a_{2} ; x_{2} D_{q}\right)_{y_{2}} F\left(a_{3} ; x_{3} D_{q}\right)_{y_{3}} \\
& \times\left\{\frac{1}{\left(y_{1} y_{2} t_{1}, y_{1} y_{2} t_{2}, y_{1} y_{3} t_{3} ; q\right)_{\infty}}\right\} \\
& =F\left(a_{1} ; x_{1} D_{q}\right)_{y_{1}} F\left(a_{2} ; x_{2} D_{q}\right)_{y_{2}} \\
& \quad \times\left\{\frac{\left(a_{3} x_{3} y_{1} t_{3} ; q\right)_{\infty}}{\left(y_{1} y_{3} t_{3}, y_{1} x_{3} t_{3} ; q\right)_{\infty}} \frac{1}{\left(y_{1} y_{2} t_{1}, y_{1} y_{2} t_{2} ; q\right)_{\infty}}\right\} \\
& =\sum_{j_{1}=0}^{\infty} \frac{\left(a_{2} ; q\right)_{j_{1}}\left(t_{1} x_{2}\right)^{j_{1}}}{(q ; q)_{j_{1}}} F\left(a_{1} ; x_{1} D_{q}\right)_{y_{1}} \\
& \times\left\{\left(y_{1}^{j_{1}}\left(a_{3} y_{1} x_{3} t_{3}, a_{2} y_{1} x_{2} t_{2} q^{j_{1}} ; q\right)_{\infty}\right)\right. \\
& \quad \times\left(\left(y_{1} y_{3} t_{3}, y_{1} x_{3} t_{3}, y_{1} y_{2} t_{1},\right.\right. \\
& \left.\left.\left.y_{1} y_{2} t_{2} q^{j_{1}}, y_{1} x_{2} t_{2} ; q\right)_{\infty}\right)^{-1}\right\} .
\end{aligned}
$$

Applying Lemma 18 and simplifying, we get the theorem. This completes the proof.

Proof of Theorems 6 and 7. Using Lemma 17, then Theorem 6 can be obtained by Lemma 20 directly.

Using Lemma 17 and then applying Lemma 18, we can get Theorem 7.

Proof of Theorem 8. LHS of (10) is equal to

$$
\begin{aligned}
& F\left(a ; x D_{q}\right)_{y}\left\{\frac{\left(y t_{1}, y t_{4} ; q\right)_{\infty}}{\left(y t_{2}, y t_{3} ; q\right)_{\infty}}\right\} \\
& =\sum_{n=0}^{\infty} \frac{(a ; q)_{n} x^{n}}{(q ; q)_{n}} D_{q}^{n}\left\{\frac{\left(y t_{1}, y t_{4} ; q\right)_{\infty}}{\left(y t_{2}, y t_{3} ; q\right)_{\infty}}\right\}
\end{aligned}
$$




$$
\begin{aligned}
& =\sum_{n=0}^{\infty} \frac{(a ; q)_{n} x^{n}}{(q ; q)_{n}} \\
& \times \sum_{k=0}^{n}\left[\begin{array}{l}
n \\
k
\end{array}\right] q^{k(k-n)} D_{q}^{k}\left\{\frac{\left(y t_{1} ; q\right)_{\infty}}{\left(y t_{2} ; q\right)_{\infty}}\right\} \\
& \times D_{q}^{n-k}\left\{\frac{\left(y t_{4} q^{k} ; q\right)_{\infty}}{\left(y t_{3} q^{k} ; q\right)_{\infty}}\right\} \\
& =\frac{\left(y t_{1}, y t_{4} ; q\right)_{\infty}}{\left(y t_{2}, y t_{3} ; q\right)_{\infty}} \sum_{n=0}^{\infty} \frac{(a ; a)_{n}\left(x t_{3}\right)^{n}}{(q ; q)_{n}} \\
& \times \sum_{k=0}^{n} \frac{(q ; q)_{n}\left(t_{2} / t_{3}\right)^{k}}{(q ; q)_{k}(q ; q)_{n-k}} \frac{\left(t_{1} / t_{2}, y t_{3} ; q\right)_{k}\left(t_{4} / t_{3} ; q\right)_{n-k}}{\left(y t_{q} ; q\right)_{k}} \\
& =\frac{\left(y t_{1}, y t_{4} ; q\right)_{\infty}}{\left(y t_{2}, y t_{3} ; q\right)_{\infty}} \sum_{j=0}^{\infty} \frac{\left(a, t_{1} / t_{2}, y t_{3} ; q\right)_{j}\left(x t_{2}\right)^{j}}{\left(q, y t_{1}, y t_{4} ; q\right)_{j}} \\
& \times{ }_{2} \Phi_{1}\left(\begin{array}{cc}
a q^{j}, & \frac{t_{4}}{t_{3}} \\
& y t_{4} q^{j}
\end{array} ; q, x t_{3}\right) ;
\end{aligned}
$$

we complete the proof.

Proof of Theorem 9. LHS of (11) equates to

$$
\begin{aligned}
& F\left(a ; x D_{q}\right)_{y} F\left(a_{0} ; x_{0} D_{q}\right)_{y_{0}} F\left(a_{1} ; x_{1} D_{q}\right)_{y_{1}} \\
& \times F\left(a_{2} ; x_{2} D_{q}\right)_{y_{2}} F\left(a_{3} ; x_{3} D_{q}\right)_{y_{3}} \\
& \times\left\{\frac{y^{s} y_{0}^{r}}{\left(y y_{0} t, y y_{1} t_{1}, y y_{2} t_{2}, y y_{3} t_{3} ; q\right)_{\infty}}\right\} \\
& =F\left(a ; x D_{q}\right)_{y} F\left(a_{0} ; x_{0} D_{q}\right)_{y_{0}} F\left(a_{1} ; x_{1} D_{q}\right)_{y_{1}} \\
& \times F\left(a_{2} ; x_{2} D_{q}\right)_{y_{2}}\left\{\frac{y^{s} y_{0}^{r}}{\left(y y_{0} t, y y_{1} t_{1}, y y_{2} t_{2} ; q\right)_{\infty}}\right. \\
& \left.\times \frac{\left(a_{3} y x_{3} t_{3} ; q\right)_{\infty}}{\left(\left(y y_{3} t_{3}, y x_{3} t_{3} ; q\right)_{\infty}\right)}\right\} \\
& =F\left(a ; x D_{q}\right)_{y} F\left(a_{0} ; x_{0} D_{q}\right)_{y_{0}} F\left(a_{1} ; x_{1} D_{q}\right)_{y_{1}} \\
& \times\left\{\frac{y^{s} y_{0}^{r}}{\left(y y_{0} t, y y_{1} t_{1} ; q\right)_{\infty}} \frac{\left(a_{2} y x_{2} t_{2} ; q\right)_{\infty}}{\left(\left(y y_{2} t_{2}, y x_{2} t_{2} ; q\right)_{\infty}\right)}\right. \\
& \left.\times \frac{\left(a_{3} y x_{3} t_{3} ; q\right)_{\infty}}{\left(\left(y y_{3} t_{3}, y x_{3} t_{3} ; q\right)_{\infty}\right)}\right\} \\
& =F\left(a ; x D_{q}\right)_{y} F\left(a_{0} ; x_{0} D_{q}\right)_{y_{0}}\left\{\frac{y^{s} y_{0}^{r}}{\left(y y_{0} t ; q\right)_{\infty}}\right\} \\
& \times\left\{\frac{\left(a_{1} y x_{1} t_{1} ; q\right)_{\infty}}{\left(\left(y y_{1} t_{1}, y x_{1} t_{1} ; q\right)_{\infty}\right)} \frac{\left(a_{2} y x_{2} t_{2} ; q\right)_{\infty}}{\left(\left(y y_{2} t_{2}, y x_{2} t_{2} ; q\right)_{\infty}\right)}\right. \\
& \left.\times \frac{\left(a_{3} y x_{3} t_{3} ; q\right)_{\infty}}{\left(\left(y y_{3} t_{3}, y x_{3} t_{3} ; q\right)_{\infty}\right)}\right\} \text {. }
\end{aligned}
$$

If we set

$$
\begin{aligned}
f(y)= & F\left(a_{0} ; x_{0} D_{q}\right)_{y_{0}}\left\{\frac{y^{s} y_{0}^{r}}{\left(y y_{0} t ; q\right)_{\infty}}\right\} \\
& \times\left\{\frac{\left(a_{1} y x_{1} t_{1} ; q\right)_{\infty}}{\left(\left(y y_{1} t_{1}, y x_{1} t_{1} ; q\right)_{\infty}\right)} \frac{\left(a_{2} y x_{2} t_{2} ; q\right)_{\infty}}{\left(\left(y y_{2} t_{2}, y x_{2} t_{2} ; q\right)_{\infty}\right)}\right. \\
& \left.\times \frac{\left(a_{3} y x_{3} t_{3} ; q\right)_{\infty}}{\left(\left(y y_{3} t_{3}, y x_{3} t_{3} ; q\right)_{\infty}\right)}\right\},
\end{aligned}
$$

using Lemma 24, we get $f(y)$; then employing Lemma 18 , we can get the desired result.

Proof of Theorem 10. LHS of (12) equates to

$$
\begin{aligned}
& F\left(a_{1} ; x_{1} D_{q}\right)_{y_{1}} F\left(a_{2} ; x_{2} D_{q}\right)_{y_{2}} F\left(a_{3} ; x_{3} D_{q}\right)_{y_{3}} \\
& \quad \times F\left(a_{4} ; x_{4} D_{q}\right)_{y_{4}}\left\{\frac{\left(t_{1} y_{2} y_{3} y_{4}, t_{4} y_{1} y_{2} y_{3} ; q\right)_{\infty}}{\left(t_{2} y_{1} y_{3} y_{4}, t_{3} y_{1} y_{2} y_{4} ; q\right)_{\infty}}\right\} ;
\end{aligned}
$$

then using Lemma 18 four times we can get the result.

\section{Some Other Cases and a Homogeneous $q$-Mehler's Formula}

In this section we obtain Mehler's formula involving a ${ }_{3} \Phi_{2}$ series and then give an interesting relationship between the polynomial multiplications.

Theorem 25 (cf. [1, Equation (1.17)] or [7, Lemma 12]). One has

$$
\begin{aligned}
& \sum_{n=0}^{\infty} \phi_{n}^{(a)}(x, y) \phi_{n}^{(b)}(X, Y) \frac{t^{n}}{(q ; q)_{n}} \\
& \quad=\frac{(a x Y t, b y X t ; q)_{\infty}}{(x Y t, y X t, y Y t ; q)_{\infty}}{ }_{3} \Phi_{2}\left(\begin{array}{ccc}
a, & b, & y Y t \\
& a x Y t, & b y X t
\end{array} ; q, x X t\right) .
\end{aligned}
$$

Proof. LHS of Theorem 25 is equal to

$$
\begin{aligned}
\sum_{n=0}^{\infty} \phi_{n}^{(b)}(X, Y) \frac{t^{n}}{(q ; q)_{n}} F\left(a ; x D_{q}\right)_{y}\left\{y^{n}\right\} \\
=F\left(a ; x D_{q}\right)_{y}\left\{\sum_{n=0}^{\infty} \sum_{k=0}^{n} \frac{(b ; q)_{k} X^{k} Y^{n-k}(t y)^{n}}{(q ; q)_{k}(q ; q)_{n-k}}\right\} \\
=F\left(a ; x D_{q}\right)_{y}\left\{\frac{(b t X y ; q)_{\infty}}{(t X y ; q)_{\infty}} \frac{1}{(t Y y ; q)_{\infty}}\right\} \\
=\frac{(a x Y t, b y X t ; q)_{\infty}}{(x Y t, y X t, y Y t ; q)_{\infty}} \\
\quad \times{ }_{3} \Phi_{2}\left(\begin{array}{c}
a, \quad b, \quad y Y t \\
a x Y t, \quad b y X t
\end{array} ;, x X t\right) .
\end{aligned}
$$

This completes the proof. 
Remark 26. Theorem 25 can be reduced to the common $q$-Mehler's formula (cf. [1, Equation (1.6)]) by setting $a=b=$ 0 and $y=Y=1$. Employing this formula, we can show that the polynomials $\Psi_{n}\left(a, b ; x_{1}, y_{1} ; z\right)$ defined by Andrews [4] can be expressed by $\phi_{n}^{(a)}(x, y)$. Using Sear's nonterminating ${ }_{3} \Phi_{2}$ transformation formula [15, page 241, Equation III.10]

$$
\begin{aligned}
& { }_{3} \Phi_{2}\left(\begin{array}{rrr}
A, & B, & C \\
D, & E
\end{array} ; q, \frac{D E}{A B C}\right) \\
& =\frac{(B, D E / A B, D E / B C ; q)_{\infty}}{(D, E, D E / A B C ; q)_{\infty}}
\end{aligned}
$$

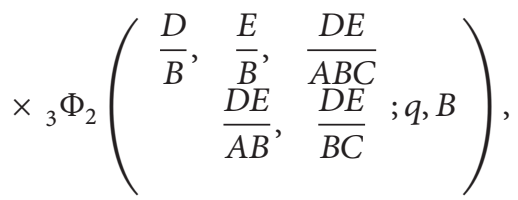

then the right-hand side of (49) reduces to

$$
\begin{aligned}
& \frac{\left(a, x y X Y t^{2}, b x X t ; q\right)_{\infty}}{(x Y t, y X t, y Y t, x X t ; q)_{\infty}} \\
& \quad \times{ }_{3} \Phi_{2}\left(\begin{array}{cc}
\frac{b y X t}{a}, & x Y t, \quad x X t \\
x y X Y t^{2}, & b x X t
\end{array} ;, a\right) .
\end{aligned}
$$

Then setting $x X=z, y X=y_{1}$, and $x Y=x_{1}$, we have (cf. [4, Theorem 4.1])

$$
\begin{aligned}
\sum_{n=0}^{\infty} \phi_{n}^{(a)}(x, y) \phi_{n}^{(b)}(X, Y) \frac{t^{n}}{(q ; q)_{n}} & \left(a, b z t, x_{1} y_{1} t^{2} ; q\right)_{\infty} \\
= & \frac{\left(t, y_{1} t, z t, x_{1} t ; q\right)_{\infty}}{\left(b t y_{1}\right.}, z t, \quad x_{1} t \\
& \times{ }_{3} \Phi_{2}\left(\begin{array}{r}
\frac{b t,}{a}, x_{1} y_{1} t^{2}
\end{array}\right) .
\end{aligned}
$$

Comparison of the above identity with Theorem 4.1 that appeared in [4] gives the following.

Corollary 27 (cf. [4, Corollary 3.1]). If $x X=z, y X=y_{1}$, and $x Y=x_{1}$, then

$$
\Psi_{n}\left(a, b ; x_{1}, y_{1} ; z\right)=\phi_{n}^{(a)}(x, y) \phi_{n}^{(b)}(X, Y) .
$$

Combined with Theorem 6 , if we replace $(x, y, X, Y, a, b, t)$ by $\left(x_{1}, y_{1}, 1, t_{2} / t_{1}, a_{1}, t_{3} / t_{1}, t_{1}\right)$, respectively, and then equate the coefficient of $t_{1}^{N}$, we have the following.
Corollary 28. One has

$$
\begin{aligned}
& \frac{\phi_{N}^{(a)}\left(x_{1}, y_{1}\right) \phi_{N}^{(b)}(1, Y)}{(q ; q)_{N}} \\
&=\sum_{m_{1}+m_{2}+m_{3}=N} \phi_{m_{1}+m_{2}+m_{3}}^{(a)}\left(x_{1}, y_{1}\right) \\
& \times \frac{Y^{m_{2}} b^{m_{3}}(-1)^{m_{3}} q^{\left(m_{3}-1\right) m_{3} / 2}}{(q ; q)_{m_{1}}(q ; q)_{m_{2}}(q ; q)_{m_{3}}} .
\end{aligned}
$$

Theorem 29 (cf. [1, Equation (1.17)]). If $m, n \in N$, then

$$
\begin{aligned}
& \sum_{m, n=0}^{\infty} \phi_{m+n}^{\left(a_{1}\right)}\left(x_{1}, y_{1}\right) \phi_{m}^{\left(a_{2}\right)}\left(x_{2}, y_{2}\right) \phi_{n}^{\left(a_{3}\right)} \\
& \times\left(x_{3}, y_{3}\right) \frac{t^{m} z^{n}}{(q ; q)_{m}(q ; q)_{n}} \frac{\left(a_{1} x_{1} x_{3} z, a_{2} x_{2} y_{1} t, a_{3} x_{3} y_{1} z ; q\right)_{\infty}}{\left(x_{1} x_{3} z, x_{2} y_{1} t, x_{3} y_{1} z, y_{1} y_{2} t, y_{1} y_{3} z ; q\right)_{\infty}} \\
& \times \sum_{j_{1}, j_{2}, j_{3}=0}^{\infty} \frac{\left(x_{1} y_{3} z\right)^{j_{1}}\left(x_{1} x_{2} t\right)^{j_{2}}\left(x_{1} y_{2} t\right)^{j_{3}}}{(q ; q)_{j_{1}}(q ; q)_{j_{2}}(q ; q)_{j_{3}}} \\
& \quad \times \frac{\left(a_{2} x_{2} / y_{2}, x_{2} y_{1} t ; q\right)_{j_{3}}\left(a_{3} x_{3} z /\left(x_{2} t\right) ; q\right)_{j_{2}}}{\left(a_{2} x_{2} y_{1} t ; q\right)_{j_{3}}} \\
& \times \frac{\left(y_{1} y_{3} z ; q\right)_{j_{2}+j_{3}}\left(a_{1}, x_{3} y_{1} z ; q\right)_{j_{1}+j_{2}+j_{3}}}{\left(a_{3} x_{3} y_{1} z ; q\right)_{j_{2}+j_{3}}\left(a_{1} x_{1} x_{3} z ; q\right)_{j_{1}+j_{2}+j_{3}}}
\end{aligned}
$$

provided that $\max \left\{\left|x_{1} y_{3} z\right|,\left|x_{3} y_{1} z\right|,\left|x_{1} y_{2} t\right|,\left|x_{2} y_{1} t\right|,\left|y_{1} y_{2} t\right|\right.$, $\left.\left|y_{1} y_{3} z\right|,\left|a_{1} x_{1} x_{3} z\right|\right\}<1$.

Proof. Applying Lemma 17 and then using q-binomial theorem, we get that the LHS of (56) comes to

$$
\begin{aligned}
& F\left(a_{1}, x_{1} D_{q}\right)_{y_{1}} F\left(a_{2}, x_{2} D_{q}\right)_{y_{2}} F\left(a_{3}, x_{3} D_{q}\right)_{y_{3}} \\
& \quad \times\left\{\frac{1}{\left(y_{1} y_{2} t, y_{1} y_{3} z ; q\right)_{\infty}}\right\} .
\end{aligned}
$$

Twice applying Lemma 22, the above identity is equal to

$$
\begin{aligned}
F\left(a_{1}, x_{1} D_{q}\right)_{y_{1}}\left\{\frac{\left(a_{2} x_{2} y_{1} t, a_{3} x_{3} y_{1} z ; q\right)_{\infty}}{\left(y_{1} y_{2} t, x_{2} y_{1} t, y_{1} y_{3} z, x_{3} y_{1} z ; q\right)_{\infty}}\right\} \\
=\sum_{n=0}^{\infty} \frac{\left(a_{1} ; q\right)_{n} x_{1}^{n}}{(q ; q)_{n}} D_{q}^{n} \\
\quad \times\left\{\frac{\left(a_{2} x_{2} y_{1} t, a_{3} x_{3} y_{1} z ; q\right)_{\infty}}{\left(y_{1} y_{2} t, x_{2} y_{1} t, y_{1} y_{3} z, x_{3} y_{1} z ; q\right)_{\infty}}\right\} .
\end{aligned}
$$

For (58), by using $q$-Leibnitz rule three times and using $q$-binomial theorem, we can achieve the proof after simplification. 
Taking $a_{1}=a_{2}=a_{3}=0, y_{1}=y_{2}=y_{3}=1$, we have the following.

Corollary 30 (cf. [9, Equation (4.4)]). One has

$$
\begin{aligned}
\sum_{m, n=0}^{\infty} H_{m+n}\left(x_{1}\right) H_{m}\left(x_{2}\right) H_{n}\left(x_{3}\right) \frac{t^{m} z^{n}}{(q ; q)_{m}(q ; q)_{n}} & \\
= & \frac{\left(x_{1} x_{3} z^{2} ; q\right)_{\infty}}{\left(x_{1} z, x_{1} x_{3} z, x_{2} t, x_{3} z, t, z ; q\right)_{\infty}} \\
& \times \sum_{j_{1}, j_{2}=0}^{\infty} \frac{\left(x_{2} t ; q\right)_{j_{2}}\left(z, x_{3} z ; q\right)_{j_{1}+j_{2}}\left(x_{1} t\right)^{j_{1}+j_{2}} x_{2}}{(q ; q)_{j_{1}}(q ; q)_{j_{2}}\left(x_{1} x_{3} z^{2} ; q\right)_{j_{1}+j_{2}}} .
\end{aligned}
$$

Remark 31. Corollary 30 is equivalent to [9, Equation (4.4)] after applying $q$-Heine transformation formula to simplify. Note that the process of proof is only using $q$-Leibnitz rule, so we can remove the restrictive condition $a=q^{-F}, b=q^{-G}$ in [7, Theorem 3]. Employing the same method, we recover Theorem 7 as follows.

Theorem 32. If $m_{i} \in N, \max \left\{\left|y t_{i}\right|,\left|x t_{i}\right|\right\}<1, i=1,2, \ldots, n$, then

$$
\begin{aligned}
& \sum_{m_{1}, \ldots, m_{n}=0}^{\infty} \phi_{m_{1}+\cdots+m_{n}}^{(a)}(x, y) \frac{t_{1}^{m_{1}} \cdots t_{n}^{m_{n}}}{(q ; q)_{m_{1}} \cdots(q ; q)_{m_{n}}} \\
& =\frac{\left(a x t_{1} ; q\right)_{\infty}}{\left(x t_{1}, y t_{1}, y t_{2}, \ldots, y t_{n} ; q\right)_{\infty}} \\
& \quad \times \sum_{j_{1}, j_{2}, \ldots, j_{n-1}=0}^{\infty} \frac{\left(a, y t_{1} ; q\right)_{j_{1}+j_{2}+\cdots+j_{n-1}} x^{j_{1}+j_{2}+\cdots+j_{n-1}}}{\left(a x t_{1} ; q\right)_{j_{1}+j_{2}+\cdots+j_{n-1}}(q ; q)_{j_{1}}(q ; q)_{j_{2}}} \\
& \quad \times\left(\left(y t_{2} ; q\right)_{j_{2}+\cdots+j_{n-1}}, \ldots,\left(y t_{n-2} ; q\right)_{j_{n-2}+j_{n-1}}\right. \\
& \left.\quad \times\left(y t_{n-1} ; q\right)_{j_{n-1}} t_{2}^{j_{1}} t_{3}^{j_{2}}, \ldots, t_{n}^{j_{n-1}}\right) \\
& \quad \times\left((q ; q)_{j_{3}}, \ldots,(q ; q)_{j_{n-1}}\right)^{-1} .
\end{aligned}
$$

Proof. Using Lemma 17, LHS of (60) is equal to

$$
\begin{aligned}
& F\left(a ; x D_{q}\right)_{y}\left\{\frac{1}{\left(y t_{n}, y t_{n-1}, \ldots, y t_{1} ; q\right)_{\infty}}\right\} \\
& =\sum_{k=0}^{\infty} \frac{(a ; q)_{k} x^{k}}{(q ; q)_{k}} D_{q}^{k}\left\{\frac{1}{\left(y t_{n}, y t_{n-1}, \ldots, y t_{1} ; q\right)_{\infty}}\right\} \\
& =\sum_{k=0}^{\infty} \frac{(a ; q)_{k} x^{k}}{(q ; q)_{k}} \\
& \quad \times \sum_{j_{1}=0}^{k}\left[\begin{array}{l}
k \\
j_{1}
\end{array}\right] q^{j_{1}\left(j_{1}-k\right)} D_{q}^{j_{1}}\left\{\frac{1}{\left(y t_{n}, y t_{n-1}, \ldots, y t_{2} ; q\right)_{\infty}}\right\} \\
& \quad \times D_{q}^{k-j_{1}}\left\{\frac{1}{\left(y t_{1}^{j_{1}} ; q\right)_{\infty}}\right\}
\end{aligned}
$$

$$
\begin{aligned}
& =\sum_{k=0}^{\infty} \frac{(a ; q)_{k} x^{k}}{(q ; q)_{k}} \\
& \times \sum_{j_{1}=0}^{k}\left[\begin{array}{l}
k \\
j_{1}
\end{array}\right] D_{q}^{j_{1}}\left\{\frac{1}{\left(y t_{n}, y t_{n-1}, \ldots, y t_{2} ; q\right)_{\infty}}\right\} \\
& \quad \times \frac{t_{1}^{k-j_{1}}\left(y t_{1} ; q\right)_{j_{1}}}{\left(y t_{1} ; q\right)_{\infty}} .
\end{aligned}
$$

Exchanging the order of summation and using $q$-binomial, theorem (61) is equal to

$$
\begin{gathered}
\frac{\left(a x t_{1} ; q\right)_{\infty}}{\left(x t_{1}, y t_{1} ; q\right)_{\infty}} \sum_{j_{1}=0}^{\infty} \frac{\left(a, y t_{1} ; q\right)_{j_{1}} x^{j_{1}}}{\left(q, a x t_{1} ; q\right)_{j_{1}}} \\
\times D_{q}^{j_{1}}\left\{\frac{1}{\left(y t_{n}, y t_{n-1}, \ldots, y t_{2} ; q\right)_{\infty}}\right\} \\
=\frac{\left(a x t_{1} ; q\right)_{\infty}}{\left(x t_{1}, y t_{1} ; q\right)_{\infty}} \sum_{j_{1}=0}^{\infty} \frac{\left(a, y t_{1} ; q\right)_{j_{1}} x^{j_{1}}}{\left(q, a x t_{1} ; q\right)_{j_{1}}} \\
\times D_{q}^{j_{1}}\left\{\frac{1}{\left(y t_{n}, y t_{n-1}, \ldots, y t_{3} ; q\right)_{\infty}}\right. \\
\left.\times \frac{1}{\left(y t_{2} ; q\right)_{\infty}}\right\} .
\end{gathered}
$$

Applying $q$-Leibnitz and exchanging the order, (62) is equal to

$$
\begin{aligned}
& \frac{\left(a x t_{1} ; q\right)_{\infty}}{\left(x t_{1}, y t_{1}, y t_{2} ; q\right)_{\infty}} \\
& \quad \times \sum_{j_{2}=0}^{\infty} \frac{\left(y t_{2} ; q\right)_{j_{2}}}{(q ; q)_{j_{2}}} D_{q}^{j_{2}}\left\{\frac{1}{\left(y t_{n}, y t_{n-1}, \ldots, y t_{3} ; q\right)_{\infty}}\right\} \\
& \quad \times \sum_{j_{1}=0}^{\infty} \frac{\left(a, y t_{1} ; q\right)_{j_{1}+j_{2}}}{(q ; q)_{j_{2}}\left(a x t_{1} ; q\right)_{j_{1}+j_{2}}} .
\end{aligned}
$$

By induction, similar proof can be performed to get the desired result.

Taking $a=0, y=1$ in (60), we have the following.

Corollary 33. If $m_{i} \in N, \max \left\{\left|t_{i}\right|,\left|x t_{i}\right|\right\}<1, i=1,2, \ldots, n$, then

$$
\begin{aligned}
& \sum_{m_{1}, \ldots, m_{n}=0}^{\infty} H_{m_{1}+\cdots+m_{n}}(x) \frac{t_{1}^{m_{1}} \cdots t_{n}^{m_{n}}}{(q ; q)_{m_{1}} \cdots(q ; q)_{m_{n}}} \\
& \quad=\frac{1}{\left(x t_{1}, t_{1}, t_{2}, \ldots, t_{n} ; q\right)_{\infty}}
\end{aligned}
$$




$$
\begin{aligned}
\times \sum_{j_{1}, j_{2}, \ldots, j_{n-1}=0}^{\infty} & \frac{\left(t_{1} ; q\right)_{j_{1}+j_{2}+\cdots+j_{n-1}} x^{j_{1}+j_{2}+\cdots+j_{n-1}}}{(q ; q)_{j_{1}}(q ; q)_{j_{2}}} \\
\times & \left(\left(t_{2} ; q\right)_{j_{2}+\cdots+j_{n-1}} \cdots\left(t_{n-2} ; q\right)_{j_{n-2}+j_{n-1}}\right. \\
& \left.\times\left(t_{n-1} ; q\right)_{j_{n-1}} t_{2}^{j_{1}} t_{3}^{j_{2}} \cdots t_{n}^{j_{n-1}}\right) \\
& \times\left((q ; q)_{j_{3}} \cdots(q ; q)_{j_{n-1}}\right)^{-1} .
\end{aligned}
$$

\section{Conflict of Interests}

The author declares that there is no conflict of interests regarding the publication of this paper.

\section{Acknowledgments}

The author is supported by Jiangsu Overseas Research and Training Program for University Prominent Young and Middle-Aged Teachers and Presidents and University National Natural Science Foundation of Jiangsu (no. 14KJB110002). The author is also supported by the National Natural Science Foundation of China (nos. 11371163 and 11001098).

\section{References}

[1] W. A. Al-Salam and L. Carlitz, "Some orthogonal qpolynomials," Mathematische Nachrichten, vol. 30, pp. 47-61, 1965.

[2] W. A. Al-Salam and T. S. Chihara, "Convolutions of orthonormal polynomials," SIAM Journal on Mathematical Analysis, vol. 7, no. 1, pp. 16-28, 1976.

[3] R. A'lvarez-Nodarse and R. S. Costas-Santos, "Factorization method for difference equations of hypergeometric type on nonuniform lattices," Journal of Physics A: Mathematical and General, vol. 34, no. 27, pp. 5551-5569, 2001.

[4] G. E. Andrews, "Carlitz and the general ${ }_{3} \varphi_{2}$," Ramanujan Journal, vol. 13, no. 1-3, pp. 311-318, 2007.

[5] D. M. Bressoud, "A simple proof of Mehler's formula for $q$ Hermite polynomials," Indiana University Mathematics Journal, vol. 29, no. 4, pp. 577-580, 1980.

[6] J. Cao, "New proofs of generating functions for Rogers-Szegö polynomials," Applied Mathematics and Computation, vol. 207, no. 2, pp. 486-492, 2009.

[7] J. Cao, "Generalizations of certain Carlitz's trilinear and Srivastava-Agarwal type generating functions," Journal of Mathematical Analysis and Applications, vol. 396, no. 1, pp. 351-362, 2012.

[8] L. Carlitz, "Some inverse relations," Duke Mathematical Journal, vol. 40, pp. 893-901, 1973.

[9] L. Carlitz, "Generating functions for certain Q-orthogonal polynomials," Collectanea Mathematica, vol. 23, no. 2, pp. 91104, 1972.

[10] W. Y. C. Chen, H. L. Saad, and L. H. Sun, "An operator approach to the Al-Salam-carlitz polynomials," Journal of Mathematical Physics, vol. 51, no. 4, Article ID 022003JMP, 2010.

[11] M. E. H. Ismail, D. Stanton, and G. Viennot, "The combinatorics of $q$-Hermite polynomials and the Askey-Wilson integral,"
European Journal of Combinatorics, vol. 8, no. 4, pp. 379-392, 1987.

[12] M. E. H. Ismail and D. R. Masson, " $q$-Hermite polynomials, biorthogonal rational functions, and q-beta integrals," Transactions of the American Mathematical Society, vol. 346, no. 1, pp. 63-116, 1994.

[13] M. E. H. Ismail and D. Stanton, "Classical orthogonal polynomials as moments," Canadian Journal of Mathematics, vol. 49, no. 3, pp. 520-542, 1997.

[14] H. M. Srivastava and V. K. Jain, "Some multilinear generating functions for q-Hermite polynomials," Journal of Mathematical Analysis and Applications, vol. 144, no. 1, pp. 147-157, 1989.

[15] G. Gasper and M. Rahman, Basic Hypergeometric Series, Cambridge University Press, Cambridge, UK, 1990.

[16] G. E. Andrews, "On the foundations of combinatorial theory. V. Eulerian differential operators," Applied Mathematics, vol. 50, pp. 345-375, 1971.

[17] W. Y. C. Chen and Z. G. Liu, "Parameter augmentation for basic hypergeometric series. II," Journal of Combinatorial Theory A, vol. 80, no. 2, pp. 175-195, 1997.

[18] J.-P. Fang, " $q$-differential operator identities and applications," Journal of Mathematical Analysis and Applications, vol. 332, no. 2, pp. 1393-1407, 2007.

[19] J.-P. Fang, "Extensions of q-Chu-Vandermonde's identity, Journal of Mathematical Analysis and Applications, vol. 339, no. 2, pp. 845-852, 2008.

[20] J.-P. Fang, "A q-differential operator identity and its applications," Journal of East China Normal University, no. 1, pp. 20-24, 2008.

[21] J. P. Fang, "Note on a q-operator identity," Ars Combinatoria, vol. 105, pp. 395-401, 2012.

[22] Z.-G. Liu, "Some operator identities and $q$-series transformation formulas," Discrete Mathematics, vol. 265, no. 1-3, pp. 119139, 2003.

[23] Z.-G. Liu, "Two $q$-difference equations and $q$-operator identities," Journal of Difference Equations and Applications, vol. 16, no. 11, pp. 1293-1307, 2010.

[24] S. Roman, "The theory of the umbral calculus. I," Journal of Mathematical Analysis and Applications, vol. 87, no. 1, pp. 58$115,1982$.

[25] S. Roman, "More on the umbral calculus, with emphasis on the q-umbral calculus," Journal of Mathematical Analysis and Applications, vol. 107, no. 1, pp. 222-254, 1985. 


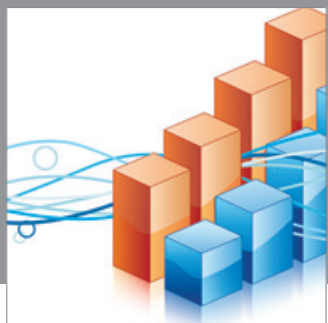

Advances in

Operations Research

mansans

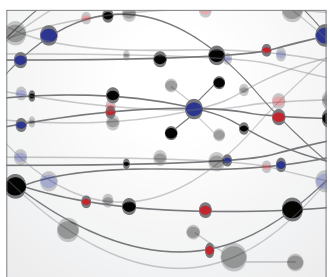

The Scientific World Journal
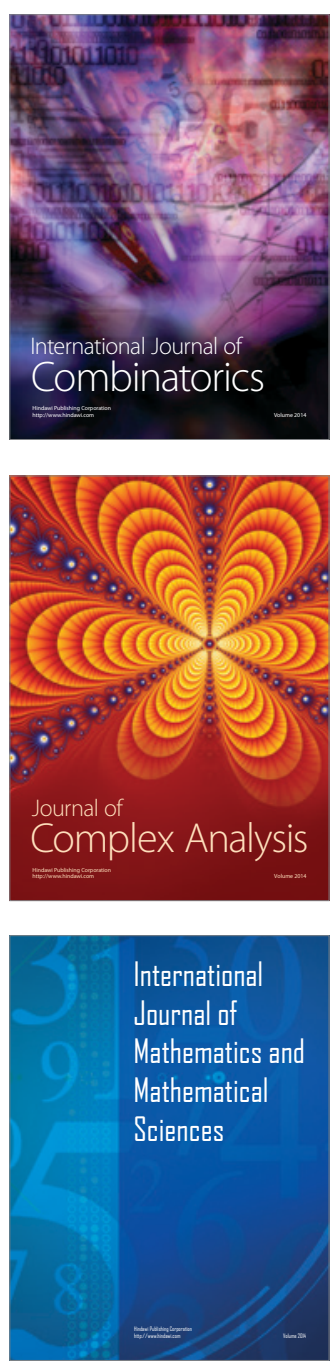
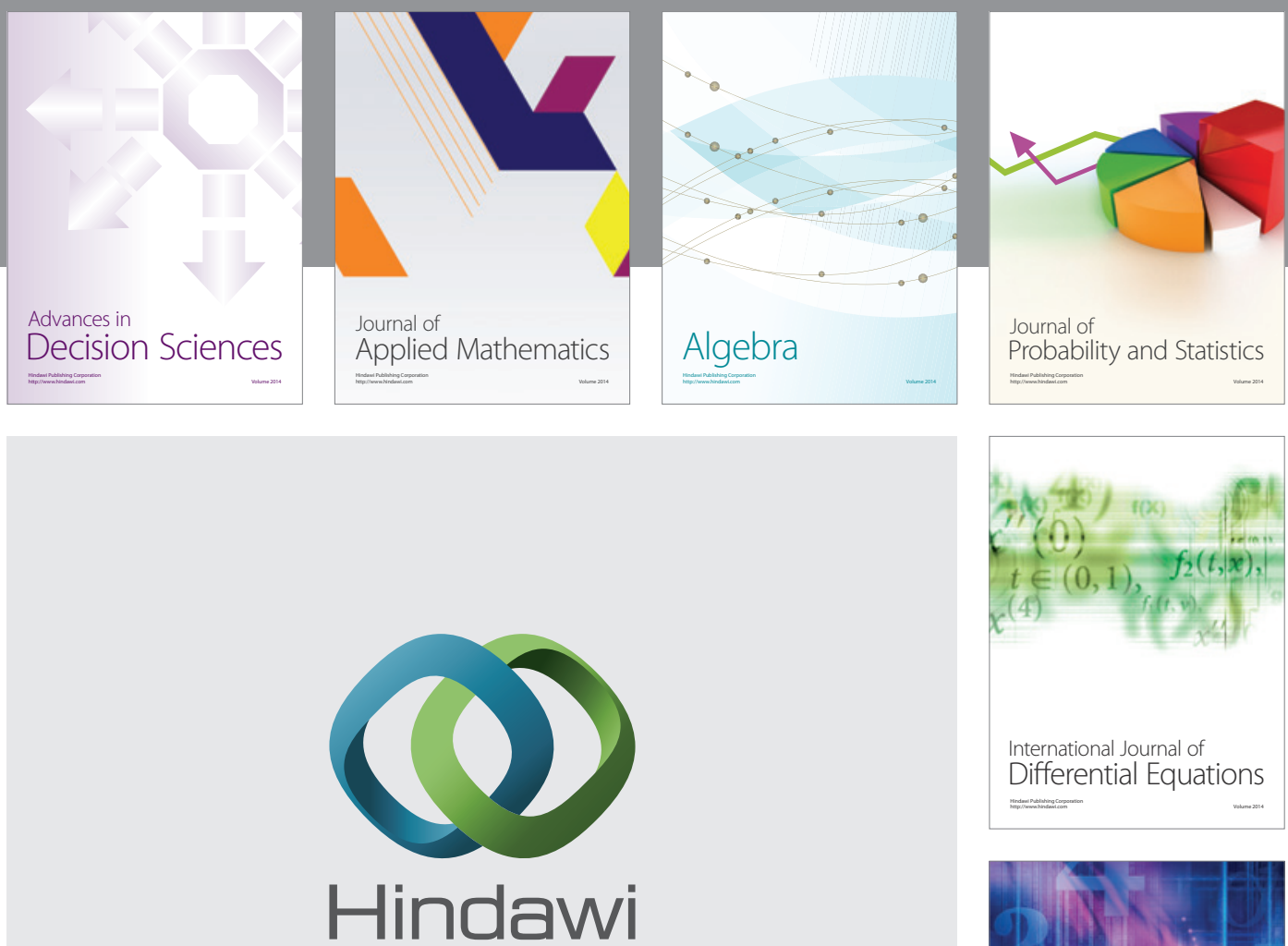

Submit your manuscripts at http://www.hindawi.com
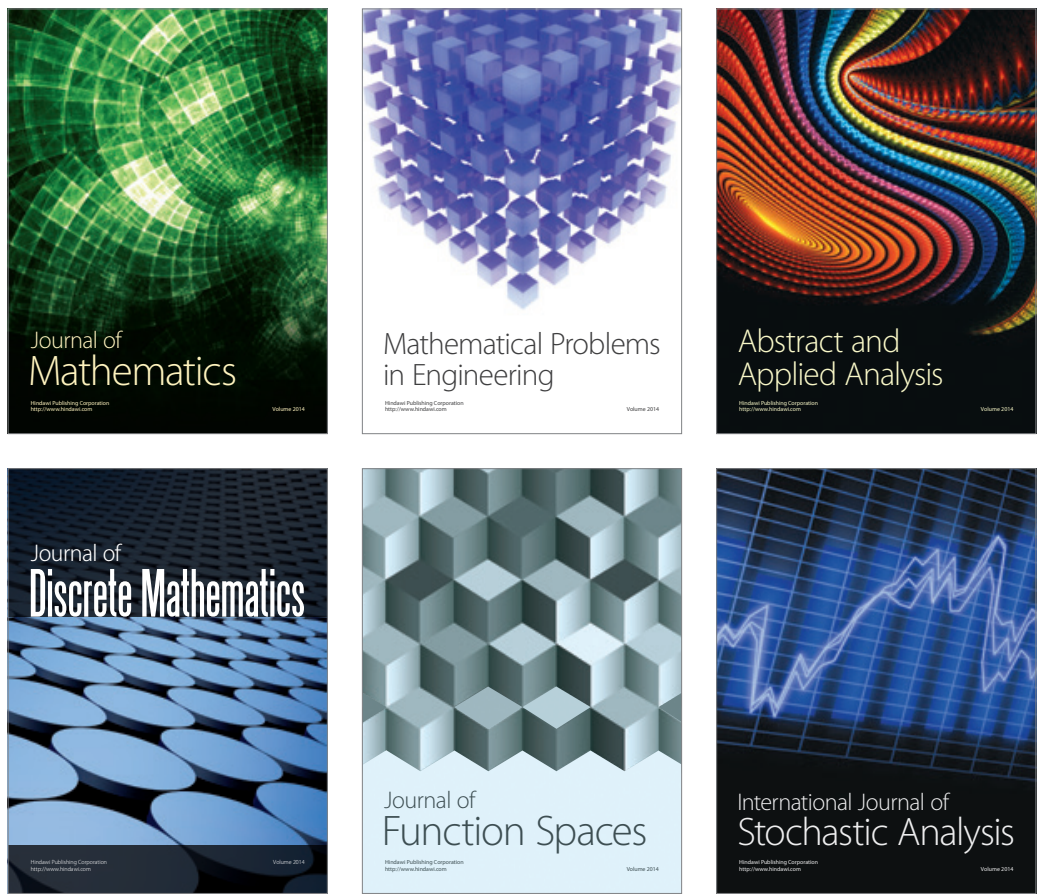

Journal of

Function Spaces

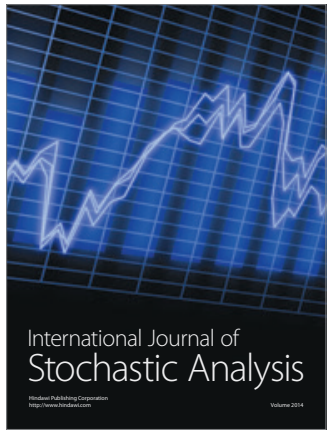

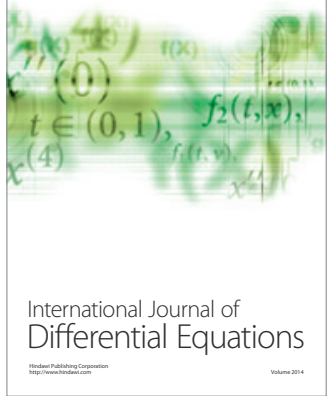
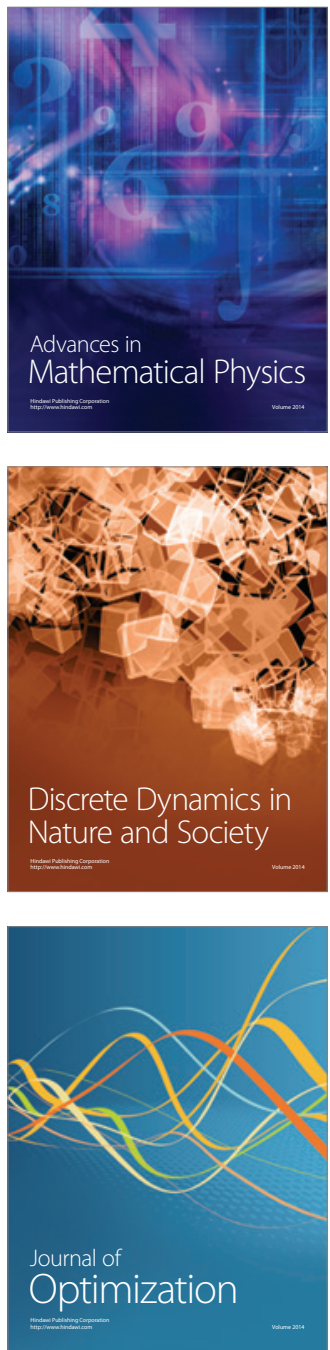